Pacific Journal of Mathematics

A GENERALIZATION OF THE UNIT INTERVAL

CORnetT 


\section{A GENERALIZATION OF THE UNIT INTERVAL}

\section{William M. CORNETte}

Convex sets are discussed here in linear spaces over scalars other than the reals. To facilitate this development, the interval $[0,1]$ is generalized to a unit interval in an arbitrary division ring. The interval $[0,1]$ is shown to be the maximum generalized unit interval in the real number field, the complex number field, and the quaternion division ring. Several elementary theorems on convexity are proved for linear spaces over scalars having generalized unit intervals of certain types.

For a real vector space $\mathscr{V}$, a set $A \subset \mathcal{V}$ is convex iff $\forall x, y \in A$, $\forall \lambda \in[0,1](1-\lambda) x+\lambda y \in A$. As might be expected, some of the vector space axioms are not needed when study is restricted to the convex subset itself. In fact, instead of the two maps:

$$
\begin{aligned}
D: \mathscr{R} \times \mathscr{V} & \rightarrow \mathscr{V} \\
(a, x) & \rightarrow a x \\
E: \mathcal{V} \times \mathscr{V} & \rightarrow \mathscr{V} \\
(x, y) & \rightarrow x+y
\end{aligned}
$$

satisfying the usual vector space axioms, all that is required is a map:

$$
\begin{aligned}
T:[0,1] \times A \times A & \rightarrow A \\
(\lambda, x, y) & \rightarrow(1-\lambda) x+\lambda y
\end{aligned}
$$

such that $\forall \lambda, \mu \in[0,1]$ and $\forall x, y, z \in A$

(1) $(1-\lambda) x+\lambda y=\lambda y+(1-\lambda) x$

(2) $(1-\lambda) x+\lambda[(1-\mu) y+\mu z]$

$$
=(1-\lambda \mu)\left[\left(1-\frac{(1-\lambda) \mu}{1-\lambda \mu}\right) x+\frac{(1-\lambda) \mu}{1-\lambda \mu} y\right]+\lambda \mu z
$$

(3) $(1-\lambda) x+\lambda x=x$

(4) $(1-\lambda) x+\lambda y=(1-\lambda) x+\lambda z$ implies $y=z$.

These axioms have been studied in some depth $[1,6]$ and have been applied to axiomatic quantum theory $[1,2,6]$. Such a development of convex structures lacks much of the generality that is possible. For example, the cardinality of points on a line segment is restricted to the cardinality of the continuum. Also, the scalars used in axiomatic quantum theory should be the complex numbers [3], or possibly the 
quaternions [4]; in these cases, it would be more convenient to treat these scalars without involving any real vector space.

2. Unit intervals. Using the axioms for the map $T$ as motivation, a definition is presented of a unit interval for an arbitrary division ring. First a quasi-unit interval $\mathscr{I}$ is a subset of the division ring $\mathscr{F}$ such that

(1) $\lambda,-\lambda \in \mathscr{I}$ implies $\lambda=0$

(2) $0,1 \in \mathscr{I}$

(3) $(1-\lambda) \mu+\lambda \nu \in \mathscr{I}$, if $\lambda, \mu, \nu \in \mathscr{I}$.

LEMMA 2.1. If $\mathscr{I}$ is a quasi-unit interval, then

(a) $1-\lambda \in \mathscr{I}$, if $\lambda \in \mathscr{I}$

(b) $\lambda \mu \in \mathscr{I}$, if $\lambda, \mu \in \mathscr{I}$

(c) $\lambda, \lambda^{-1} \in \mathscr{I}$ implies $\lambda=1$.

Proof. Parts (a) and (b) are trivial. For part (c), assume $\lambda, \lambda^{-1} \in$ $\mathscr{I}$. Then $1-\lambda \in \mathscr{I}$ and $1-\lambda^{-1} \in \mathscr{I}$, so that

$$
\lambda\left(1-\lambda^{-1}\right)=\lambda-1=-(1-\lambda) \in \mathscr{I} ;
$$

therefore $1-\lambda=0$ and $\lambda=1$.

In this light it seems natural to define an interval $[a, b]_{\mathscr{F}}$ to be

$$
\{c \in \mathscr{F}:(1-\lambda) a+\lambda b=c \text {, for some } \lambda \in \mathscr{I}\} \text {. }
$$

The subscript $\mathscr{I}$ may be omitted if it is understood in the context of the material.

The development to this point closely parallels the approach of Green and Gustin to quasi-convex sets in linear spaces [5]. Unfortunately, to properly develop a convex structure, this is not quite enough. It is necessary to define the natural ordering of the quasi-unit interval as $\lambda \leqq \mu$ iff $\exists \sigma \in \mathscr{I}$ such that $\sigma \mu=\lambda$. The following lemma is immediate.

LEMMA 2.2 .

(a) $\lambda \leqq \mu$ and $\mu \leqq \lambda$ iff $\lambda=\mu$.

(b) $\lambda \leqq \mu$ and $\mu \leqq \nu$ implies $\lambda \leqq \nu$.

(c) $\forall \lambda \in \mathscr{I}, 0 \leqq \lambda \leqq 1$.

(d) $\lambda \leqq \mu$ iff $\lambda \mu^{-1} \in \mathscr{I}$.

From part (d) above, it immediately follows that:

THEOREM 2.3. I is totally ordered iff either $\lambda \mu^{-1} \in \mathscr{I}$ or $\mu \lambda^{-1} \in \mathscr{I}$ whenever $\lambda, \mu \in \mathscr{I}$. 
This theorem motivates the definition of a unit interval as a quasi-unit interval such that $\lambda \mu^{-1} \in \mathscr{I}$ or $\mu \lambda^{-1} \in \mathscr{I}$ whenever $\lambda, \mu \in \mathscr{I}$. Henceforth, when $[\mu, \nu]_{\mathscr{\rho}}$ is written, it will be understood that $\mu \leqq \nu$.

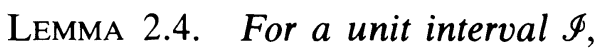

(a) $[0, \mu]=\{\nu \in \mathscr{I}: \nu \leqq \mu\}$

(b) $[\mu, 1]=\{\nu \in \mathscr{I}: \mu \leqq \nu\}$

(c) $[\mu, \nu]=\{\lambda \in \mathscr{I}: \mu \leqq \lambda \leqq \nu\}$.

Proof. Part (a) is immediate.

(b) If $\nu \in[\mu, 1]$, then $\exists \lambda \in \mathscr{I}$ such that $(1-\lambda) \mu+\lambda=\nu$.

$$
\mu[1-(1-\lambda)(1-\mu)]^{-1}=\mu[(1-\lambda) \mu+\lambda]^{-1} \in \mathscr{I}
$$

implies $\nu \geqq \mu$. But $\nu \geqq \mu$ implies $\mu \nu^{-1} \in \mathscr{I}$ and $\nu-\mu \in \mathscr{I}$, as $\nu-\mu=$ $\left(1-\mu \nu^{-1}\right) \nu$, so that $\nu \leqq 1$ and $\nu-\mu \leqq 1-\mu$ imply

$$
(\nu-\mu)(1-\mu)^{-1} \in \mathscr{I} .
$$

Thus

$$
\left[1-(\nu-\mu)(1-\mu)^{-1}\right] \mu+(\nu-\mu)(1-\mu)^{-1}=\nu .
$$

(c) If $\lambda \in[\mu, \nu]$, then $\exists \sigma \in \mathscr{I}$ such that $\lambda=(1-\sigma) \mu+\sigma \nu$. Therefore it is sufficient to show and

(i) $[(1-\sigma) \mu+\sigma \nu] \nu^{-1} \in \mathscr{I}$

(ii) $\mu[(1-\sigma) \mu+\sigma \nu]^{-1} \in \Phi$.

Part (i) is clear, since $(1-\sigma) \mu \nu^{-1}+\sigma \in\left[\mu \nu^{-1}, 1\right]$. Part (ii) follows from (i) and (b), which show that $\mu \nu^{-1} \leqq(1-\sigma) \mu \nu^{-1}+\sigma$.

COROLLARY 2.5. If $\lambda \leqq \mu_{0}$ and $\mu \in\left[\mu_{0}, \mu_{1}\right]$ for any $\mu_{1} \geqq \mu_{0}$, then $\lambda \leqq \mu$.

COROllary 2.6. $\lambda \leqq \mu$ iff $(1-\mu) \leqq(1-\lambda)$.

Proof. By Lemma 2.4, if $\lambda \leqq \mu$, then $\exists \rho, \tau \in \Phi$ such that $\mu=\rho \lambda$ and $\lambda=(1-\tau) \mu+\tau$. Solving for $\tau$, one obtains

$$
\tau=(\lambda-\mu)(1-\mu)^{-1}=1-(1-\lambda)(1-\mu)^{-1}
$$

therefore $(1-\lambda)(1-\mu)^{-1} \in \mathscr{I}$. The converse follows similarly.

In light of Lemma 2.4 , it seems reasonable to define $(\mu, \lambda)=$ $[\mu, \lambda]-\{\mu, \lambda\} . \quad[\mu, \lambda)$ and $(\mu, \lambda]$ are defined analogously. 
There are two topologies to be considered on $\mathscr{I}$. The first is the natural order topology, generated by the closed sets $[\lambda, \mu]_{0}=\{\nu: \lambda \leqq \nu \leqq$ $\mu\}$ for $\lambda, \mu \in \mathscr{I}$ and $\lambda \leqq \mu$. The second topology is the closed interval topology, generated by the closed sets

$$
[\lambda, \mu]_{\mathscr{I}}=\{\nu \in \mathscr{I}: \nu=(1-\sigma) \lambda+\sigma \mu, \text { for some } \sigma \in \mathscr{I}\}
$$

for $\lambda, \mu \in \mathscr{I}$. By the use of the above theorems, one immediately obtains the following:

COROLlaRY 2.7. The closed interval topology is finer than the natural order topology for a quasi-unit interval.

COROLlARY 2.8. The closed interval topology coincides with the natural order topology for a unit interval.

Now consider a unit interval $\mathscr{I}$ of an infinite division ring $\mathscr{F}$ and the sequence $\left\{\lambda^{n}\right\}$, where $\lambda \in \mathscr{I}$. Trivially, $\lambda^{n} \leqq \lambda^{n-1}, \forall n \in \mathcal{N}(\mathcal{N}$ is the set of positive integers). If $\lambda^{n}$ converges in the natural order topology, which is clearly Hausdorff, to a value $\mu \in \mathscr{I}$, then $\mu \leqq \lambda^{k}, \forall k \in \mathcal{N}$, so that $\mu \lambda^{-n} \leqq \lambda^{m}, \forall m, n \in \mathcal{N}$. But $\mu \leqq \mu \lambda^{-m} \leqq \lambda^{n} \quad \forall m, n \in \mathcal{N}$ implies $\mu=$ $\mu \lambda^{-m}$. Indeed, if $\mu<\mu \lambda^{-m}$, then $\exists p \in \mathcal{N}$ such that $\mu \leqq \lambda^{p} \leqq \mu \lambda^{-m}$. Contradiction. Thus $\mu\left(1-\lambda^{m}\right)=0$, so $\lambda=1$ or $\mu=0$. If $\lambda=1$, clearly $\mu=1$. Moreover, where $0 \neq \lambda \neq 1,\left\{\lambda^{n}\right\}$ is a strictly decreasing sequence with a lower bound, so it does converge. Therefore, the following has been proved:

LEMMA 2.9. For an element $\lambda \neq 1$ of a unit interval in an infinite division ring, $\lambda^{n} \rightarrow 0$ as $n \rightarrow \infty$ in the natural order topology.

The major scalars of interest in physical theory are the rationals, the reals, the complex numbers, and the quaternions, denoted by $\mathscr{K}, \mathscr{R}, \mathscr{C}$, and 2 , respectively. The unit intervals as they are normally defined in the rational and the real fields obviously form maximal generalized unit intervals under set inclusion in their respective fields. The same is also true of the complex numbers, as shown below.

Consider $\mathscr{F}=\mathscr{C}$ and let $[0,1] \subset \mathscr{I}$. Then $\mathrm{re}^{i \pi / 2}, \mathrm{re}^{i \pi}$, and $\mathrm{re}^{i 3 \pi / 2}(r \neq 0)$ are not in $\mathscr{I}$, and $r e^{i 0}, r>1$, is not in $\mathscr{I}$. Also, as $\mathscr{I}$ is connected in the sense of $\mathscr{C} \cong \mathscr{R}^{2}$, the second and third quadrants do not contain elements in $\mathscr{I}$. Then by the fact that $\lambda^{n} \in \mathscr{I}$ if $\lambda \in \mathscr{I},[0,1]=\mathscr{I}$. By an analogous argument, the same is true of the quaternion division ring $\mathscr{Q}$.

For any division ring there exists a quasi-unit interval, namely the set $\{0,1\}$. This unit interval is the trivial unit interval $\mathscr{I}_{0}$. Some other examples of unit intervals are given as follows: 
(1) $\Phi_{\mathscr{R}}=[0,1]$

(2) $\mathscr{I}_{\mathscr{K}}=[0,1] \cap \mathscr{K}$

(3) $\mathscr{I}_{\mathscr{L}}=[0,1] \cap \mathscr{L}$ for any subfield $\mathscr{L}$ of $\mathscr{R}$ containing $\mathscr{K}$.

Lemma 2.10. If $\mathscr{F}$ is a finite division ring, then $\mathscr{I}=\{0,1\}$.

Proof. For $\lambda \in \mathscr{I}-\{0,1\}$, there exists a prime $p$ such that $\lambda^{p}=$ 1. Then $\lambda^{p-1}=\lambda^{-1} \in \mathscr{I}$. Thus $\lambda=1$. Contradiction.

From Lemma 2.10 , it immediately follows that Lemma 2.9 is trivial for a finite division ring.

THEOREM 2.11. If $\mathscr{I}$ is a nontrivial unit interval, then $(1+1)^{-1} \in \mathscr{I}$.

Proof. First note that $(1+1)^{-1}$ exists, since $\mathscr{I}$ is nontrivial, and $\mathscr{F}$ is therefore infinite. Next assume $\lambda \in \mathscr{I}-\mathscr{I}_{0}$. Then

$$
(1-\lambda) \lambda+\lambda(1-\lambda)=(1+1) \lambda(1-\lambda) \in \mathscr{I}
$$

and

$$
(1-\lambda) \lambda+\lambda 0=\lambda(1-\lambda) \in \mathscr{I} .
$$

Total ordering implies either $\lambda \mu^{-1} \in \mathscr{I}$ or $\mu \lambda^{-1} \in \mathscr{I}, \quad \forall \lambda, \mu \in$ $\mathscr{I}$. Therefore $(1+1) \in \mathscr{I}$ or $(1+1)^{-1} \in \mathscr{I}$; but if $(1+1) \in \mathscr{I}$, then 1 $(1+1)=-1 \in \mathscr{I}$. Contradiction. Therefore, $(1+1)^{-1} \in \mathscr{I}$.

LEMMA 2.12. If $\mathscr{I}$ is nontrivial, then $\mathscr{I}_{\mathscr{K}} \subset \mathscr{I}$.

Proof. $\forall \lambda, \mu \in \mathscr{I} \lambda \mu \leqq \lambda$, so

$$
(1-\lambda)(1-\lambda \mu)^{-1} \in \mathscr{I}
$$

Thus

$$
(1-\lambda)\left(1-\lambda^{2}\right)^{-1} \in \mathscr{I}
$$

implies $(1+\lambda)^{-1} \in \mathscr{I}$. If $m^{-1} \in \mathscr{I}$ for a natural number $m$, then $(m+1)^{-1} \in \mathscr{I}$. Since $2^{-1} \in \mathscr{I}$ by Theorem 2.11, $m^{-1} \in \mathscr{I}, \quad \forall m \in$ $\mathcal{N}$. Thus $n m^{-1} \in \mathscr{I}$ for all natural numbers $n \leqq m$, by total ordering.

By an argument similar to the above showing that $\mathscr{I}_{\mathscr{R}}$ is a maximal unit interval in $\mathscr{C}$, it is easy to show that $\mathscr{C}-\mathscr{I}$ is dense in $\mathscr{C}-\mathscr{I}_{\mathscr{R}}$ and $\mathscr{I} \cap \mathscr{I}_{\mathscr{R}}$ is dense in $\mathscr{I}_{\mathscr{R}}$ for any nontrivial unit interval $\mathscr{I}$. By an analogous argument, the same is true of the quaternions.

Now if the completion $\overline{\mathscr{I}}$ of $\mathscr{I}$ in the reals, the complex numbers, or the quaternions is also a unit interval, then $\mathscr{I}_{\mathscr{R}}$ is the maximum unit interval for these fields. Obviously $0,1 \in \overline{\mathscr{I}}$ and if the sequences $\left\{\lambda_{n}\right\}$, 
$\left\{\mu_{n}\right\}$, and $\left\{\sigma_{n}\right\}$ in $\mathscr{I}$ converge to $\lambda, \mu$, and $\sigma$, respectively, in $\bar{\Phi}$, then obviously

$$
\left(1-\lambda_{n}\right) \mu_{n}+\lambda_{n} \sigma_{n} \rightarrow(1-\lambda) \mu+\lambda \sigma \in \overline{\mathscr{I}}
$$

Assume all elements of the sequences $\left\{\lambda_{n}\right\}$ and $\left\{\mu_{n}\right\}$ are nonzero. If the sequence $\left\{\lambda_{n} \mu_{n}^{-1}\right\}$ is partitioned into the subsequences $\left\{\lambda_{n} \mu_{n}^{-1}: \lambda_{n} \mu_{n}^{-1} \in \mathscr{I}\right\}$ and $\left\{\lambda_{n} \mu_{n}^{-1}: \lambda_{n} \mu_{n}^{-1} \notin \mathscr{I}\right\}$, then one of these subsequences will converge. Both will converge if and only if $\lambda=\mu$. If the first subsequence converges then $\lambda \mu^{-1} \in \overline{\mathscr{I}}$. If not, then $\mu \lambda^{-1} \in \overline{\mathscr{I}}$. Finally, assume $\mu=-\lambda$. Then choose the subsequence which converges. Assume that it is the first subsequence. Then

$$
\lambda_{n} \mu_{n}^{-1} \rightarrow \lambda(-\lambda)^{-1}=-1 \in \bar{\Phi} .
$$

Contradiction. Therefore, the following theorem has been proven:

THEOREM 2.13. If $\mathscr{I}$ is a unit interval in a division ring $\mathscr{F}$ with a norm and $\bar{\Phi}$ is the completion of $\mathscr{I}$ in the norm-induced topology, then $\bar{\Phi}$ is also a unit interval.

From the denseness arguments given above, it follows that:

COROLlaRy 2.14. $\Phi_{\mathscr{R}}$ is the maximum unit interval in the reals, the complexes, and the quaternions.

3. Convex sets. Using the concept of a unit interval $\mathscr{I}$ developed above, a set $A$ in a linear space $\mathscr{V}$ over a division ring $\mathscr{F}$ is convex (with respect to $\mathscr{I}$ ) iff $(1-\lambda) x+\lambda y \in A \forall x, y \in A$ and $\forall \lambda \in$ $\mathscr{I}$. It is readily apparent that if $\mathscr{F}$ is finite, any subset of $\mathscr{V}$ is convex. Similarly if $\mathscr{I}$ is trivial for an infinite division ring, any subset of $\mathscr{V}$ is convex. If the scalars are the rationals and $\mathscr{I}$ is nontrivial, then the convex sets are the usual ones; subsets of $\mathscr{R}, \mathscr{C}$, and $\mathscr{2}$ that are convex with respect to any nontrivial unit interval have closures that are convex in the usual sense.

To demonstrate the generality of this approach to convexity, a few standard terms are defined and a few basic theorems are stated below.

THEOREM 3.1. If $A$ and $B$ are nondisjoint sets convex with respect to the unit interval $\mathscr{I}$, then $A \cap B$ is also convex with respect to $\mathscr{I}$.

The proof of this is trivial. It is assumed that the empty set is not convex. 
THEOREM 3.2. Let $x_{1}, x_{2}, \cdots, x_{n} \in A$, where $A$ is convex with respect to the nontrivial unit interval $\mathscr{I}$. Then for $\lambda_{1}, \lambda_{2}, \cdots, \lambda_{n} \in \mathscr{I}$ such that

$$
\begin{aligned}
& \sum_{i=1}^{n} \lambda_{l}=1, \\
& \sum_{i=1}^{n} \lambda_{i} x_{i} \in A .
\end{aligned}
$$

Before this proof is given, it is necessary to show that for any $1 \leqq j \leqq n$

$$
\lambda_{l}\left(\sum_{i=1}^{n-1} \lambda_{l}\right)^{-1} \in \mathscr{I} \text { and }\left(\sum_{i=1}^{n-1} \lambda_{l}\right)^{-1} \lambda_{j} \in \mathscr{I}
$$

provided the sum in parentheses is nonzero. Assume that $\lambda_{\imath} \in \mathscr{I}-\mathscr{I}_{0}$ $(i=1, \cdots, n-1)$. Recall from the proof of Lemma 2.12 that $\lambda \in \mathscr{I}$ implies $(1+\lambda)^{-1} \in \mathscr{I}$. Similarly, $\lambda(1+\lambda)^{-1}=\left(1+\lambda^{-1}\right)^{-1} \in \mathscr{I}$. Now proceeding by induction, for $n=3$,

$$
\lambda_{1}\left(\lambda_{1}+\lambda_{2}\right)^{-1}=\left(1+\lambda_{2} \lambda_{1}^{-1}\right) \in \mathscr{I}
$$

by the total ordering of $\mathscr{I}$, and similarly for $\lambda_{2}\left(\lambda_{1}+\lambda_{2}\right)^{-1}$. Assuming that the above holds for $m$, it suffices to show that

$$
\lambda_{m}\left(\sum_{i=1}^{m} \lambda_{i}\right)^{-1} \in \mathscr{I}
$$

This follows since

$$
\begin{aligned}
\lambda_{m}\left(\sum_{i=1}^{m} \lambda_{l}\right)^{-1} & =\lambda_{m}\left(\lambda_{m}+\sum_{i=1}^{m-1} \lambda_{i}\right)^{-1} \\
& =\left(1+\left(\lambda_{m-1}\left(\sum_{i=1}^{m-1} \lambda_{i}\right)^{-1}\right)^{-1} \lambda_{m-1} \lambda_{m}^{-1}\right) \in \mathscr{I}
\end{aligned}
$$

The remainder follows similarly. The proof of Theorem 3.2 now follows:

Proof. If $n=2$, one simply obtains $\left(1-\lambda_{2}\right) x_{1}+\lambda_{2} x_{2}$ since $\lambda_{1}=$ $1-\lambda_{2}$. For arbitrary $n$, if

$$
\sum_{i=1}^{n-1} \lambda_{l} \neq 0
$$


then

$$
\sum_{i=1}^{n} \lambda_{i} x_{\imath}=\left(1-\lambda_{n}\right) \sum_{i=1}^{n-1}\left[\lambda_{i} x_{i}\left(\sum_{\imath=1}^{n-1} \lambda_{\iota}\right)^{-1}\right]+\lambda_{n} x_{n}
$$

The theorem is then proved by induction.

Let the set $A$ be convex with respect to a nontrivial unit interval $\mathscr{I}$. A point $x_{0}$ is a boundary point of $A$ if there exist two other points $x_{1}$ and $y_{1}$ such that

$$
\left\{z=(1-\lambda) x_{1}+\lambda x_{0}: \lambda \in \mathscr{I}-\mathscr{I}_{0}\right\} \subset A
$$

and

$$
\left\{z=(1-\lambda) y_{1}+\lambda x_{0}: \lambda \in \mathscr{I}-\mathscr{I}_{0}\right\} \cap A=\varnothing .
$$

If $\mathscr{I}$ is trivial, boundary points are not defined. A boundary point may or may not be an element of $A$. The set of all boundary points of $A$ is the boundary of $A$ and is denoted $\partial A$.

Lemma 3.3. If $A$ is a convex set then $A+x$ is also convex. If the unit interval is nontrivial, then $\partial(A+x)=\partial A+x$.

This lemma follows immediately from the fact that $(1-\lambda) x+\lambda y+$ $z=(1-\lambda)(x+z)+\lambda(y+z)$.

Extending the concept of a natural order to all the scalars, an element $\alpha$ of the scalars is defined to be greater than zero if either $\alpha$ or $\alpha^{-1}$ is in $\mathscr{I}$. This is equivalent to requiring the existence of some $\lambda \in \mathscr{I}-\mathscr{I}_{0}$ such that $\lambda \alpha \in \mathscr{I}$. Also define $\alpha>\beta$ iff $(\alpha-\beta)>0$.

A sublinear functional is a function $p$ from $\mathscr{V}$ into $\mathscr{F}$ such that

(1) $p(x+y) \leqq p(x)+p(y) \forall x, y \in \mathscr{V}$

(2) $p(\alpha x)=\alpha p(x) \forall \alpha>0$ and $\forall x \in \mathscr{V}$.

THEOREM 3.4. Let $I$ be a unit interval that defines the convex sets of the linear space and an ordering on the scalars. (a) If $p$ is a sublinear functional, then for arbitrary $c \in \mathscr{F}$ and $a \in \mathscr{V}$,

$$
A=\{x: p(x-a) \leqq c\}
$$

is convex with respect to $\mathscr{I}$. and $\mathscr{I}$ is nontrivial, then

(b) If $c>0$, the range of $p$ is totally ordered, 


$$
\partial A=\{x: p(x-a)=c\}
$$

Proof. By Lemma 3.3 it is sufficient to prove the theorem for $a=0$. (a) follows from the fact that

$$
\begin{aligned}
p((1-\lambda) x+\lambda y) & \leqq(1-\lambda) p(x)+\lambda p(y) \\
& \leqq(1-\lambda) c+\lambda c=c
\end{aligned}
$$

To prove (b), assume $p\left(x_{0}\right)=c$ and let $\alpha \in \mathscr{I}-\mathscr{I}_{0}$ and $\beta>1$. For $\lambda \in \mathscr{I}, 1-(1-\alpha) \lambda \in \mathscr{I}$ and $[1+(\beta-1) \lambda]^{-1} \in \mathscr{I}$, so

$$
p\left((1-\lambda) x_{0}+\lambda \alpha x_{0}\right)<c
$$

and

$$
p\left((1-\lambda) x_{0}+\lambda \beta x_{0}\right)>c .
$$

Let $\alpha x_{0}=x_{1}$ and $\beta x_{0}=y_{1}$ in the definition of a boundary point. $\quad x_{0}$ is a boundary point.

Conversely, let $x_{0}$ be a boundary point of $A$. For $x_{1}$ and $y_{1}$ satisfying the conditions in the definition of a boundary point

$$
(1-\lambda) p\left(x_{0}\right)+\lambda p\left(x_{1}\right) \leqq c \leqq(1-\lambda) p\left(x_{0}\right)+\lambda p\left(y_{1}\right) .
$$

By letting $\lambda$ approach zero, $p\left(x_{0}\right)=c$.

A topology is convex compatible if the convergence of a sequence $\left\{\lambda_{n}\right\}$ in $\mathscr{I}$ to $\lambda \in \mathscr{I}$ implies

$$
\left(1-\lambda_{n}\right) x+\lambda_{n} y \rightarrow(1-\lambda) x+\lambda y \quad \forall x, y \in \mathscr{V}
$$

THEOREM 3.5. Let $\mathscr{V}$ be a linear space with a convex compatible topology. If $A$ is a closed convex set with respect to a nontrivial unit interval, then $\partial A \subset A$.

Proof. By Lemma $2.91-\lambda^{n} \rightarrow 1$. Let $x_{0}$ be a boundary point of $A$ and $x_{1} \in A$ such that

$$
\left\{z=(1-\lambda) x_{0}+\lambda x_{1}\right\} \subset A .
$$

Then the points $z_{n}=\left(1-\lambda^{n}\right) x_{0}+\lambda^{n} x_{1}$ approach $x_{0}$. Since $A$ is closed, $x_{0} \in A$.

It should be noted that the natural order topology on $\Phi_{\mathscr{R}}$ is 
metrizable. Let $A$ be a subset of $\mathscr{V}$ that is convex with respect to a complete unit interval $\mathscr{I}$. If there exists a topology on $\mathscr{V}$ such that $\mathscr{V}$ is a Hausdorff topological linear space and $A$ is bounded, then there exists a map

$$
\rho: A \times A \rightarrow \mathscr{I}
$$

such that

$$
\begin{gathered}
\rho(x, y)=\rho(y, x) \\
\rho(x, z) \leqq \rho(x, y)+\rho(y, z) \\
\rho(x, y)=0 \quad \text { iff } \quad x=y \quad[1,2,6] .
\end{gathered}
$$

Furthermore, $\rho(x, z)=\rho(x, y)+\rho(y, z)$ iff there exists $\mu \in \mathscr{I}$ such that $y=(1-\mu) x+\mu z$. If $A$ is not bounded, then the "only if" portion of the third condition doesn't necessarily hold. Any map satisfying these three conditions is called a generalized metric, and for any complete unit interval, the natural order topology is metrizable.

4. Conclusions. The concept of convexity is quite general. With this generalization of the unit interval, the assumption that the set of scalars is $\mathscr{R}$ can be dropped from many major theorems on convexity. In fact, it would be quite interesting to see how the theory of convexity can be developed using a generalized unit interval. Use of this generalization would tend to emphasize the actual requirements (completeness, total ordering, existence of a norm or metric, etc.) on the scalars and the unit interval needed for each theorem and would lead to better comprehension of the theory of convexity.

Acknowledgements. The author thanks R. Jamison and S. P. Gudder for several fruitful discussions on this subject.

\section{REFERENCES}

1. W. M. Cornette, Convex Pre-structures and the Mixture of Quantum States, Ph.D. Dissertation, Univ. of Denver (1973).

2. W. M. Cornette and S. P. Gudder, The mixture of quantum states, J. Math. Phys., 15 (1974), 842-850.

3. P. A. M. Dirac, The Principles of Quantum Mechanics, 4th ed., Oxford Press, London, 1958.

4. D. Finkelstein, J. M. Jauch, S. Schimonowich, and D. Speiser, Foundations of quaternionic quantum mechanics, J. Math. Phys., 3 (1962), 207-220. 
5. J. W. Green and W. Gustin, Quasiconvex sets, Canad. J. Math., 2 (1950), 489-507.

6. S. P. Gudder, Convex structures and operational quantum mechanics, Comm. Math. Phys., 29 (1973), 249-264.

Received May 10, 1974 and in revised form June 7, 1976.

Systems Development Department

Naval Weapons Center

China lake, California, 93555 



\section{PACIFIC JOURNAL OF MATHEMATICS}

\section{EDITORS}

RICHARI) ARENS (Managing Editor)

University of California

Los Angeles, CA 90024

R. A. Beaumiont

University of Washington

Seattle, WA 98105

\section{J. DugunduI}

Department of Mathematics University of Southern California Los Angeles, CA 90007

D. Gilbarg and J. Milgram Stanford University

Stanford, CA 94305

\section{ASSOCIATE EDITORS}

E. F. BECKENBACH
B. H. NEUMANN

F. Wolf

K. YoshidA

\section{SUPPORTING INSTITUTIONS}

UNIVERSITY OF BRITISH COLUMBIA CALIFORNIA INSTITUTE OF TECHNOLOGY

UNIVERSITY OF CALIFORNIA

MONTANA STATE UNIVERSITY

UNIVERSITY OF NEVADA

NEW MEXICO STATE UNIVERSITY

OREGON STATE UNIVERSITY

UNIVERSITY OF OREGON

OSAKA UNIVERSITY

\author{
UNIVERSITY OF SOUTHERN CALIFORNIA \\ STANFORD UNIVERSITY \\ UNIVERSITY OF HAWAII \\ UNIVERSITY OF TOKYO \\ UNIVERSITY OF UTAH \\ WASHINGTON STATE UNIVERSITY \\ UNIVERSITY OF WASHINGTON \\ AMERICAN MATHEMATICAL SOCIETY
}

The Supporting Institutions listed above contribute to the cost of publication of this Journal, but they are not owners or publishers and have no responsibility for its contents or policies.

Mathematical papers intended for publication in the Pacific Journal of Mathematics should be in typed form or offset-reproduced (not dittoed), double spaced with large margins. Underline Greek letters in red, German in green, and script in blue. The first $p: 1<$ graph or two must be capable of being used separately as a synopsis of the entire paper. Items of the biblography should not be cited there unless absolutely necessary, in which case they must he identified by author and Journal, rather than by item number. Manuscripts, in duplicate, may be sent to any one of the four editors. Please classify according to the scheme of Math. Reviews, Index to Vol. 39. All other communications should be addressed to the managing editor, or Elaine Barth, University of California, Los Angeles, California, 90024.

100 reprints are provided free for each article, only if page charges have been substantially paid. Additional copies may be obtained at cost in multiples of 50 .

The Pacific Journal of Mathematics is issued monthly as of January 1966. Regular subscription rate: $\$ 72.00$ a year (6 Vols., 12 issues). Special rate: $\$ 36.00$ a year to individual members of supporting institutions.

Subscriptions, orders for back numbers, and changes of address should be sent to Pacific Journal of Mathematics, 103 Highland Boulevard, Berkeley, California, 94708.

PUBLISHED BY PACIFIC JOURNAL OF MATHEMATICS, A NON-PROFIT CORPORATION Printed at Jerusalem Academic Press, POB 2390, Jerusalem, Israel.

\section{Copyright (C) 1976 Pacific Journal of Mathematics} All Rights Reserved 


\section{Pacific Journal of Mathematics}

\section{Vol. 66, No. 2 December, 1976}

Gerald A. Beer, Tax structures whose progressivity is inflation neutral..... 305

William M. Cornette, A generalization of the unit interval............. 313

David E. Evans, Unbounded completely positive linear maps on

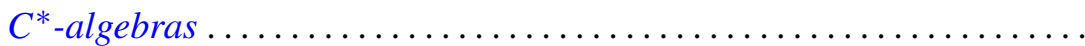

Hector O. Fattorini, Some remarks on convolution equations for

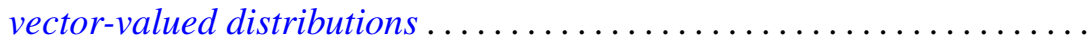

Amassa Courtney Fauntleroy, Automorphism groups of unipotent groups of

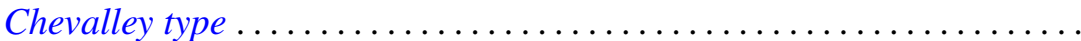

Christian C. Fenske and Heinz-Otto Peitgen, On fixed points of zero index in

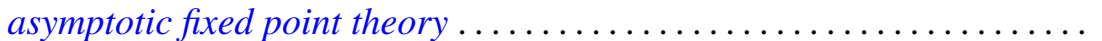

Atsushi Inoue, On a class of unbounded operator algebras. II ............

Herbert Meyer Kamowitz, The spectra of endomorphisms of algebras of

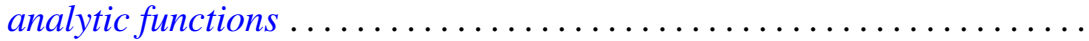

Jimmie Don Lawson, Embeddings of compact convex sets and locally compact cones ....................................

William Lindgren and Peter Joseph Nyikos, Spaces with bases satisfying certain order and intersection properties .....................

Emily Mann Peck, Lattice projections on continuous function spaces ...... 477

Morris Marden and Peter A. McCoy, Level sets of polynomials in $n$ real variables...

Francis Joseph Narcowich, An imbedding theorem for indeterminate Hermitian moment sequences......................

John Dacey O'Neill, Rings whose additive subgroups are subrings ...

Chull Park and David Lee Skoug, Wiener integrals over the sets bounded by sectionally continuous barriers .....................

Vladimir Scheffer, Partial regularity of solutions to the Navier-Stokes equations.

Eugene Spiegel and Allan Trojan, On semi-simple group algebras. II

Katsuo Takano, On Cameron and Storvick's operator valued function space integral 